\title{
Factores socioacadémicos, estilo de aprendizaje, nivel intelectual y su relación con el rendimiento académico previo de médicos internos de pregrado
}

\author{
J.L. Padierna-Luna, J. Oseguera-Rodríguez, N. Gudiño-Hernández
}

Introducción. El aprendizaje es una actividad compleja, en la que intervienen factores individuales, sociales-culturales y académicos. Objetivo. Describir factores socioacadémicos, estilos de aprendizaje, nivel intelectual y su relación con el rendimiento académico previo (promedio) de los médicos internos de pregrado (MIP). Sujetos y métodos. Se realizó una encuesta transversal analítica a MIP aplicando tres cuestionarios: datos socioacadémicos, cuestionario CHAEA (estilos de aprendizaje) y test de Raven para adultos (nivel intelectual). La muestra incluyó 174 alumnos procedentes de nueve universidades, tres privadas ( $n=43 ; 24,7 \%$ ) y seis públicas ( $n=131 ; 75,29 \%$ ). Se utilizó estadística descriptiva y regresión múltiple para establecer asociaciones entre variables. Resultados y conclusiones. Predominó el género femenino, con el $59,2 \%(n=103)$, frente al masculino, con el $40,8 \%$ ( $n$ =71). El promedio de edad fue de 23,63 años, con un rango de 21 a 33. No hubo diferencias significativas de género en el rendimiento previo $(8,21$ frente a 8,25$)$. Se midió la relación entre los factores socioacadémicos, estilos de aprendizaje y nivel intelectual con el rendimiento académico, con un intervalo de confianza del 95\%. De los datos socioacadémicos, sólo la edad se relaciona inversamente con el rendimiento, con $r=0,2$ y $p<0,001$. El estilo de aprendizaje no guarda ninguna relación con el rendimiento. El nivel intelectual se relaciona significativamente con el rendimiento $(p<0,001)$.

Palabras clave. Factores socioacadémicos. Médico interno de pregrado. Nivel intelectual. Rendimiento académico.
Socioacademic factors, style of learning, intellectual level and their relationship with the previous academic yield of medical students

Introduction. The learning is a complex activity, in that takes part individual factors, social and academic factors, among others. Aim. To describe socioacademic factors, styles of learning (SL), intellectual level (IL) and its relation with previous academic yield (academic average) of the Pre-degree interns (PDI). Subjects and methods. A cross-sectional and analytic survey to $P D I$ was applied with three questionnaires: socioacademic data, adult questionnaire CHAEA (SL) and test Raven (IL). The sample included 174 students coming from nine universities, three private $(n=43 ; 24.7 \%)$ and six public ( $\mathrm{n}=131 ; 75.29 \%)$. It was used descriptive statistic and multiple regression to look for associations between the variables. Results and conclusions. The feminine sex with the $59.2 \%$ predominated $(n=103)$ on the masculine one, $40.8 \%$ $(n=71)$. The average of age was of 23.63 years with a rank from 21 to 33. There were no significant differences of gender in the previous yield ((8.21 vs 8.25). The relation between the socioacademic factors, styles of learning and intellectual level with the academic yield was evaluated, with an interval of confidence of $95 \%$. Of the socioacademic factor, only the age is related inversely to the yield, with $r=0.2$ and $p<0.001$. The SL does not bear any relation to the yield. IL it is related significantly to the yield $(\mathrm{p}<0.001)$.

Key words. Intellectual level. Pre-degree interns. Socioacademic factors. Yield academic.
Departamento de Educación e Investigación. Hospital General de Zona n.o 4. IMSS. Celaya, Gto., México

Correspondencia Dr. José Luis Padierna Luna. Departamento de Educación e Investigación. Hospital General de Zona n. 4. Diego Rivera y Mutualismo. Colonia Suiza. Celaya, Gto.,

CP 38060, México.

Fax

$01-461-61-5-08-70$

E-mail

jlpadierna@prodigy.net.mx 


\section{Introducción}

El rendimiento académico es un indicador del aprendizaje alcanzado por el alumno; da cuenda de su trayectoria escolar hasta un momento determinado. Si la medida del rendimiento es adecuada, expresa el nivel de conocimientos previos que posee el alumno. Para algunos autores, el rendimiento previo es un predictor del éxito académico [1].

En el aprendizaje intervienen múltiples variables: factores individuales (estilo de aprender, nivel intelectual, etc.) y socioacadémicos (posición económica, escolaridad de los padres, programas académicos, etc.). Del mismo modo que hay diferentes estilos de liderazgo, de autoridad o de enseñar, hay diferentes estilos de aprender, tanto en niños como en adultos. No todos aprendemos de la misma manera ni a la misma velocidad. El estilo, en el terreno educativo, significa los indicadores cognitivos, afectivos y fisiológicos, relativamente estables, de cómo los alumnos perciben, interaccionan y responden a sus ambientes de aprendizaje. Es el modo en que el individuo aprende y que se refleja en sus diferentes habilidades, intereses, debilidades y fortalezas académicas [2].

Del aprendizaje existen tantos conceptos como teorías: conductistas, cognitivas, andragógicas, humanistas, constructivistas, etc. [1,3]. Gardner, en la teoría de inteligencias múltiples (lingüística, musical, logicomatemática, personal, interpersonal, etc.), señala que el proceso enseñanza-aprendizaje debe enfocarse a la inteligencia particular de cada persona, y el aprendizaje no es sólo el procesamiento de la información, sino que el alumno sea capaz de crear un producto [4]. Para los expertos de la UNESCO (1972), aprender es 'aprender a ser', y es una necesidad a lo largo de toda la vida [5]. Aprender a aprender significa el conocimiento y destreza necesaria para aprender con efectividad en cualquier escenario clínico en que el médico interno se encuentre; para tal propósito, sería útil al alumno conocer su propio estilo de aprendizaje, para que pueda elaborar su propio aprendizaje.

Un estilo de aprendizaje es la forma en que la mente procesa la información, y se ha estudiado por varios autores, destacadamente por Kolb [6]. El modelo en el que se basa este autor es en el aprendizaje experiencial, con cuatro estilos de aprendizaje: acomodador, divergente, asimilador y convergente, modificados por
Mumford-Honey en activo, reflexivo, teórico y pragmático, respectivamente [1]. Kolb define el aprendizaje experiencial como 'un proceso por medio del cual el conocimiento se crea a través de la transformación de la experiencia. El modelo es dinámico, en espiral o circular, y comienza como sigue en un continuum: la experiencia inmediata o concreta (etapa 1, 'siento') conduce a observaciones y reflexiones (etapa 2, 'observo'). Estas observaciones se asimilan o sintetizan dentro de conceptos (etapa 3, 'pienso'), con implicaciones para la acción (etapa 4, 'actúo'). Además, estos estilos son dialécticos. Kolb explica que las personas naturalmente prefieren ciertos estilos de aprendizaje. La preferencia por un estilo es realmente el producto de dos variables que están en conflicto y que podemos elegir; nuestro estilo de aprendizaje es producto de las siguientes decisiones:

- ¿Cómo enfocamos la tarea o atrapamos la experiencia? Preferimos hacer, ser activos (experiencia concreta) u observar, ser teóricos (conceptualización abstracta).

- ¿Cuál es nuestra respuesta emocional a la experiencia o cómo transformamos la experiencia? Preferimos pensar, ser reflexivos (observación reflexiva) o sentir, ser pragmáticos (experimentación activa).

En otras palabras, nosotros elegimos nuestro enfoque a la tarea o experiencia al optar por 1 o 2 (o soy activo o teórico, pero no ambas a la vez) y, al mismo tiempo, elegimos una respuesta emocional para transformar la experiencia en algo importante o útil al optar por 1 o 2 (o soy reflexivo o pragmático; pero no ambas a la vez) [7]. En este estudio se utilizarán los estilos de aprendizaje modificados por Mumford-Honey en virtud de que ya se han aplicado a estudiantes universitarios de habla castellana [2]. Ahora bien, la mayoría de los estudiantes universitarios tiene, en mayor o menor proporción, características de los cuatro estilos, pero para aquéllos que tienen alta preferencia por un estilo determinado de aprendizaje (superior a la media), ésa es su preferencia. Ningún estilo es correcto o incorrecto ni uno es mejor que otro. Idealmente, para que un alumno aprenda con eficacia en cualquier escenario, debe tener fortalecidos los cuatro estilos. De tal manera que conocer su estilo de aprendizaje es conocer sus fortalezas y limitaciones, con 
el propósito de que los docentes lo retroalimenten y orienten para que explote su estilo natural de aprender, pero también para que se le asesore en ejercitar otros estilos, dado que, aunque relativamente estables, son modificables y se puede aumentar el rendimiento académico [8].

Pero el aprendizaje es más complejo e influyen otros factores, como la inteligencia y aspectos socioacadémicos. De la primera existen varias definiciones y pruebas para medirla. Una prueba que mide la capacidad intelectual, independientemente de los factores culturales (razonamiento no verbal), es la prueba de matrices progresivas de Raven. Esta prueba tiene otros atributos, como medir las relaciones lógicas de los elementos abstractos, capacidad de comprensión y, lo más importante, traducir habilidades cognitivas en la lógica hipotético-deductiva [9].

Dentro de los objetivos que persigue el programa académico de internado de pregrado, entre otros, están el desarrollo de competencias clínicas a través de la integración de conocimientos, habilidades y destrezas adquiridos en años previos, $y$ el fortalecimiento de actitudes y valores propios del ejercicio de la medicina, así como el desarrollo de un pensamiento científico hipotético-deductivo para la integración diagnóstico-terapéutica en los diferentes escenarios: comunitario, clínico o quirúrgico, y de acuerdo con el modelo natural (Leavell-Clark). Éste debería complementarse con un modelo cultural de la enfermedad, dada la elevada prevalencia de padecimientos crónicos [10]. Del rendimiento de diferentes grupos de estudiantes de medicina se puede observar que, compartiendo texto y contexto, unos alumnos aprenden mejor que otros; o bien, un mismo alumno obtiene una calificación alta en un escenario clínico y baja en otro. Existen varias respuestas a esta situación, dada la complejidad del aprendizaje, pero una de ellas es que los estilos de aprender de los alumnos originan diferentes respuestas y comportamientos ante el aprendizaje.

En nuestro país no hay estudios sobre estilos de aprendizaje. En España se han estudiado en el personal de enfermería [11]. Se ha validado el cuestionario CHAEA en diversos estudiantes universitarios [12]. En este estudio se determinaron los factores socioacadémicos, los estilos de aprendizaje y el nivel intelectual de los médicos internos de pregrado (MIP) y su relación con el rendimiento académico previo.

\section{Sujetos y métodos}

Se realizó un estudio transversal analítico a estudiantes de medicina que cursaron el año de internado de pregrado, en los meses de noviembre y diciembre de 2005 y 2006, adscritos a los hospitales del sector público: Hospital General de Zona n. ${ }^{\circ} 4$ de Celaya y Hospital Regional n. ${ }^{\circ} 1$ de Querétaro del Instituto Mexicano del Seguro Social (IMSS), Hospital General de Celaya del Instituto de Seguridad y Servicios Sociales a los Trabajadores del Estado (ISSSTE), y Hospital General de la Secretaría de Salud de Celaya (SSG). La muestra incluyó 174 alumnos procedentes de nueve universidades, tres privadas $(n=43$; $24,7 \%)$ y seis públicas ( $n=131 ; 75,29 \%)$. Se eliminaron cuestionarios incompletos, dudosos o ilegibles. En condiciones óptimas, individualmente o en grupo, desayunados, descansados, a primera hora de la mañana, en un lugar tranquilo (biblioteca o auditorio) y previa explicación de las características del estudio, se aplicaron los siguientes instrumentos.

\section{Datos socioacadémicos}

Universidad de procedencia, género, edad, profesión o escolaridad de los padres, número de hermanos, estado civil, promedio global de la carrera de medicina y materias con mayor y menor calificación (se cotejaron con la cartilla de calificaciones), y especialidad que piensan estudiar (en su caso), ubicándolas posteriormente en área clínica, quirúrgica u otras. De acuerdo con el currículum de cada escuela, las materias de la carrera de medicina se clasificaron arbitrariamente en predominantemente teóricas (fisiología, embriología, gastroenterología, etc.); predominantemente prácticas (laboratorios y prácticas, cirugía y materias afines, como urología, etc.); y sociohumanistas (introducción a la clínica, bioética, historia y filosofía de la medicina, medicina preventiva, etc.).

\section{Cuestionario CHAEA sobre los estilos de aprendizaje (Tabla I)}

Este cuestionario consta de 80 ítems de respuesta dicotómica (positivo + o negativo -), si se está más de acuerdo o en desacuerdo con el ítem, respectivamente; de ellos, 20 corresponden a cada estilo de aprendizaje, distribuidos aleatoriamen- 


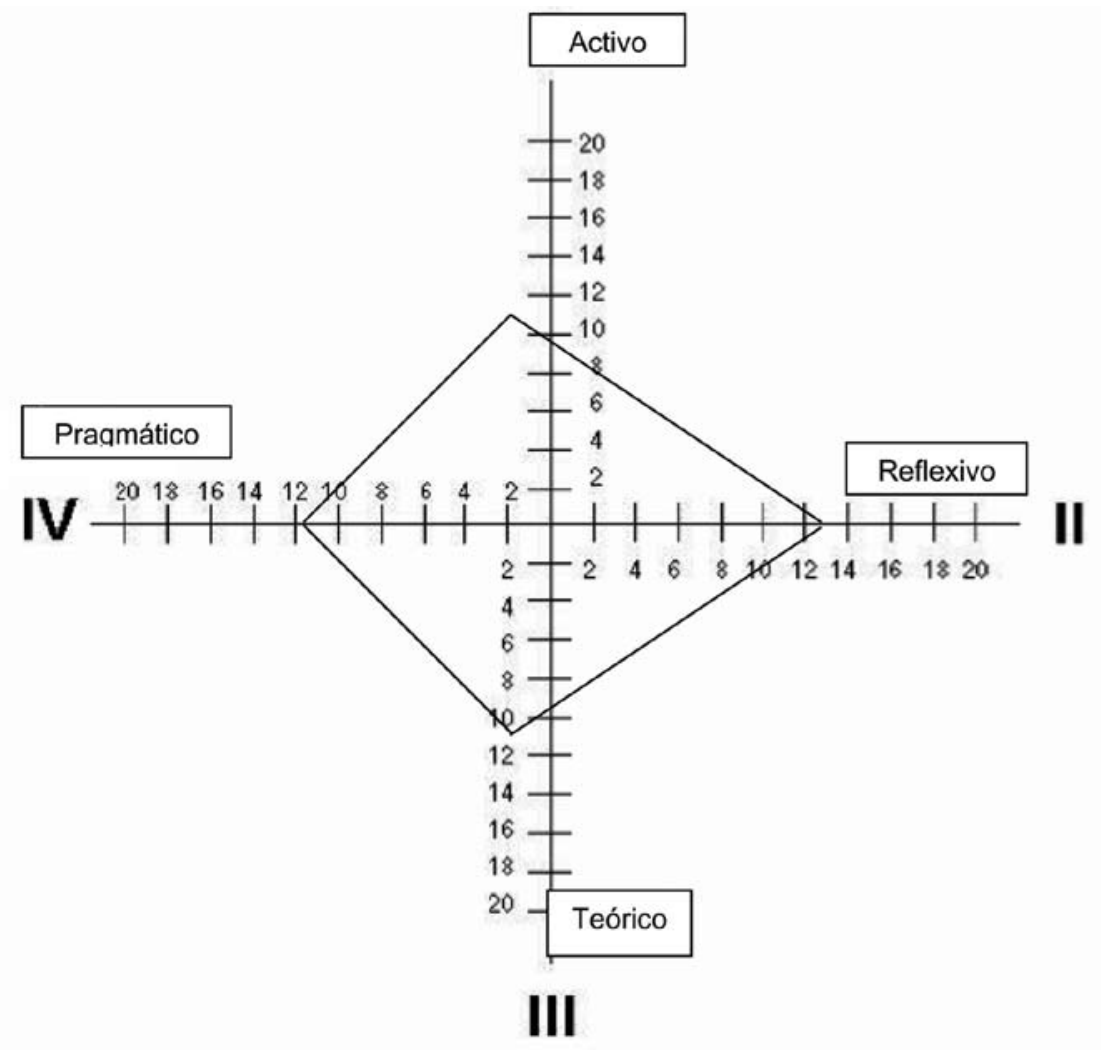

Figura 1. Gráfica de estilos de aprendizaje.

te, de tal manera que el alumno no puede distinguir a qué estilo corresponde cada pregunta. La puntuación absoluta que cada sujeto obtiene indica el nivel que alcanza en cada uno de los cuatro estilos y se anota en un gráfico (Fig. 1). De esta forma, se obtiene el estilo de aprendizaje. Cada estilo tiene sus características propias y un punto de corte medio, por debajo del cual están las preferencias bajas o muy bajas, y por arriba del cual están las preferencias altas o muy altas, de acuerdo con un baremo general (Tabla II). Para el estilo activo y teórico, el punto de corte fue de 11; para el estilo reflexivo, de 15; y para el estilo pragmático, de 12. Cada estilo posee características propias, a grandes rasgos (Tabla III).

\section{Test de matrices progresivas de Raven para adultos}

Consta de 60 problemas que se presentan en blanco y negro, distribuidos en cinco series, A, B, C,
D y E, cada una de las cuales consta, a su vez, de 12 problemas, ordenados en grado creciente de dificultad. El sujeto tiene que elegir, entre seis opciones, la imagen que concuerda con la matriz. En total son 60 y se calificó de acuerdo con una escala general para adultos. Se diagnosticó la capacidad intelectual según la puntuación, el percentil y ajustado a la edad del sujeto, de acuerdo con un baremo o carpeta de evaluación (Tabla IV). Se tomó en cuenta el tiempo de reacción del sujeto ante la prueba, según la siguiente escala: 1-15 minutos, 1630 minutos, 31-45 minutos y más de 45 minutos.

Para la variable dependiente rendimiento académico, se consideró el promedio de todas las calificaciones de los MIP desde el inicio de la carrera hasta su llegada al ciclo académico de internado de pregrado, de acuerdo con la cartilla oficial emitida por la universidad de procedencia.

En la presente investigación se plantearon tres hipótesis nulas, suponiendo una hipótesis alternativa diferente a Ho: 
Tabla I. Cuestionario Honey-Alonso de estilos de aprendizaje (fragmento).

1. Tengo fama de decir lo que pienso claramente y sin rodeos

2. Estoy seguro de lo que es bueno y lo que es malo, lo que está bien y lo que está mal

3. Muchas veces actúo sin mirar las consecuencias

4. Normalmente trato de resolver los problemas metódicamente y paso a paso

5. Creo que los formalismos coartan y limitan la actuación libre de las personas

6. Me interesa saber cuáles son los sistemas de valores de los demás y con qué criterios actúan

7. Pienso que el actuar intuitivamente puede ser siempre tan válido como actuar reflexivamente

8. Creo que lo más importante es que las cosas funcionen

9. Procuro estar al tanto de lo que ocurre aquí y ahora

10. Disfruto cuando tengo tiempo para preparar mi trabajo y realizarlo a conciencia

11. Estoy a gusto siguiendo un orden, en las comidas, en el estudio, haciendo ejercicio regularmente

12. Cuando escucho una nueva idea, en seguida comienzo a pensar cómo ponerla en práctica

13. Prefiero las ideas originales y novedosas, aunque no sean prácticas

14. Admito y me ajusto a las normas sólo si me sirven para lograr mis objetivos

15. Normalmente encajo bien con personas reflexivas, y me cuesta sintonizar con personas demasiado espontáneas, imprevisibles

16. Escucho con más frecuencia que hablo

17. Prefiero las cosas estructuradas a las desordenadas

Instrucciones para responder al cuestionario: este cuestionario ha sido diseñado para identificar su estilo de aprendizaje preferido. No es un test de inteligencia, ni de personalidad. No hay límite de tiempo para contestar el cuestionario. No le ocupará más de 15 minutos. No hay respuestas correctas o erróneas. Será útil en la medida que sea sincero/a en sus respuestas. Si está más de acuerdo que en desacuerdo con el ítem, seleccione 'Más (+)'; si, por el contrario, está más en desacuerdo que de acuerdo, seleccione 'Menos (-)'. Por favor, conteste a todos los ítems. Para facilitar el análisis del grupo, le rogamos que responda también a las preguntas de índole socioacadémica.
- No existe correlación estadísticamente significativa entre los factores socioacadémicos y el rendimiento académico.

- No existe correlación estadísticamente significativa entre el estilo de aprendizaje y el rendimiento académico.

- No existe correlación estadísticamente significativa entre el nivel intelectual (medido por el test de Raven) con el rendimiento académico.

Una vez recolectada la información, se capturó en una base de datos en el programa SPSS, versión 12 en español, para su tabulación y análisis. Se utilizó estadística descriptiva para el manejo de variables sociodemográficas, estilos de aprendizaje y nivel intelectual. Se efectuó regresión múltiple para establecer la relación entre las variables independientes con el rendimiento académico.

\section{Resultados}

La muestra incluyó a 174 alumnos procedentes de nueve universidades, tres privadas $\mathrm{A}(n=$ $12), \mathrm{B}(n=17)$ y $\mathrm{C}(n=14)$ que aportaron en su conjunto el $24,71 \%$ de la matrícula, y el resto $(n=131,75,29 \%)$ procedente de universidades públicas D, E, F, G, H e I (Tabla V). Durante el internado hubo baja de dos alumnas por problemática familiar. Predominó el género femenino, con el 59,2\% $(n=103)$, sobre el masculino, con el $40,8 \%(n=71)$. El promedio de edad fue de 23,63 años, con una rango de 21 a 33 .

Los factores socioacadémicos se muestran en la tabla VI, donde se observa que la mayoría de los alumnos son solteros, tienen padres profesionales y menos de dos hermanos. Las mejores calificaciones fueron en materias teóricas, seguidas de las prácticas y, al final, de las sociohumanistas. La inmensa mayoría desearía estudiar una especialidad y tiene discreta preferencia por las áreas quirúrgicas sobre las clínicas. El rendimiento académico previo medido a través del promedio global de calificación fue de 8,23 , con un rango de 6,9 a 9,49 y desviación estándar (DE) de 0,49. El promedio académico del sexo femenino fue de 8,21 , con una DE de 0,47 , y del género masculino de 8,25 , con una DE de 0,51 . Se midió la relación entre los factores socioacadémicos, estilos de aprendizaje y nivel intelectual con el rendi- 
Tabla II. Baremo general de las preferencias y características de estilos de aprendizaje.

\begin{tabular}{|c|c|c|c|c|c|}
\hline \multirow[b]{2}{*}{ Estilos } & \multicolumn{5}{|c|}{ Preferencia } \\
\hline & Muy baja & Baja & Moderada & Alta & Muy alta \\
\hline Activo & $0-6$ & $7-8$ & $9-12$ media $(10,7)$ & $13-14$ & $15-20$ \\
\hline Reflexivo & $0-10$ & $11-13$ & $14-17$ media $(15,37)$ & $18-19$ & 20 \\
\hline Teórico & $0-6$ & $7-9$ & $10-13$ media $(11,3)$ & $14-15$ & $16-20$ \\
\hline Pragmático & $0-8$ & $9-10$ & $11-13$ media $(12,1)$ & $14-15$ & $16-20$ \\
\hline
\end{tabular}

Tabla III. Características de los estilos de aprendizaje.

\begin{tabular}{|c|c|}
\hline $\begin{array}{l}\text { Activo } \\
\text { (improvisador) }\end{array}$ & $\begin{array}{l}\text { Se implican plenamente y sin prejuicios en tareas nuevas, aquí y ahora: animadores, arriesgados, } \\
\text { espontáneos. Improvisadores, descubridores, creativos, protagonistas, líderes, competitivos, } \\
\text { divertidos }\end{array}$ \\
\hline $\begin{array}{l}\text { Reflexivo } \\
\text { (analítico) }\end{array}$ & $\begin{array}{l}\text { Prudentes, analizan desde diferentes perspectivas. Observan, escuchan y actúan. Son distantes. } \\
\text { Son ponderados, lentos, receptivos, exhaustivos, pacientes, cuidadosos, indagadores }\end{array}$ \\
\hline $\begin{array}{l}\text { Teórico } \\
\text { (metódico } \\
\text { y objetivo) }\end{array}$ & $\begin{array}{l}\text { Elaboran teorías, son perfeccionistas, sintéticos. Profundos en su pensamiento: racionalidad } \\
\text { y objetividad. Metódicos, críticos, disciplinados, sistemáticos, relacionados, buscadores de } \\
\text { hipótesis, teorías, modelos, el porqué }\end{array}$ \\
\hline $\begin{array}{l}\text { Pragmático } \\
\text { (práctico y realista) }\end{array}$ & $\begin{array}{l}\text { Aplicación práctica de las ideas. Actúan rápidamente y con seguridad en proyectos que les } \\
\text { atraen. Si funciona es bueno. Prácticos, directos, realistas, rápidos, decididos, claros, positivos, } \\
\text { solucionadores de problemas }\end{array}$ \\
\hline
\end{tabular}

miento académico previo (promedio global de la carrera), con un intervalo de confianza del $95 \%$. De los factores socioacadémicos, sólo la edad se relaciona significativa e inversamente con el rendimiento, con $r=0,2$ y $p<0,001$ (a menor edad de estos grupos, mayor rendimiento); el estilo de aprendizaje no guarda ninguna relación con el rendimiento y el nivel intelectual se relaciona significativamente con el promedio $(p<0,001)$ (Tabla VII y Fig. 2).

Respecto a los estilos de aprendizaje, los MIP tienen preferencia alta o muy alta por el estilo teórico, seguido del pragmático, reflexivo y activo (Tabla
VIII). No hubo diferencia entre el estilo de aprendizaje con el género y escuela de procedencia.

El nivel de inteligencia, medido a través del test de Raven, tuvo una curva de distribución normal y se encontró que globalmente los MIP tienen un nivel intelectual superior al término medio $(60,9 \%)$ (Tabla IX). La inmensa mayoría de los alumnos respondió el test en un lapso entre 16 a 45 minutos (tiempo de reacción).

En conclusión, se aceptan las hipótesis nulas en el sentido de que los factores socioacadémicos (con excepción de la edad) y los estilos de aprendizaje no se relacionan con el rendimiento 
Tabla IV. Diagnóstico de la capacidad intelectual.

\begin{tabular}{|c|c|c|c|}
\hline Puntuación & Percentil & Rango & Diagnóstico \\
\hline & 95 & । & Superior (1) \\
\hline \multirow[t]{2}{*}{ Igual o superior a } & 90 & $\|+$ & \multirow{2}{*}{ Superior al termino medio (2) } \\
\hline & 75 & $\|$ & \\
\hline Superior a & 50 & $\| I+$ & Término medio (3) \\
\hline Igual a & 50 & III & Término medio (3) \\
\hline \multirow[t]{2}{*}{ Inferior a } & 50 & III- & Término medio (3) \\
\hline & 25 & IV+ & Inferior al término medio (4) \\
\hline \multirow[t]{2}{*}{ Igual o menor a } & 10 & IV & \multirow{2}{*}{ Deficiente (5) } \\
\hline & 5 & V & \\
\hline
\end{tabular}

\begin{tabular}{|llll|}
\hline \multicolumn{4}{l}{$\begin{array}{l}\text { Tabla V. Universidad de procedencia de } \\
\text { médicos internos de pregrado, promoción } \\
\text { 2005-2006. }\end{array}$} \\
\hline Universidad & Frecuencia & $\%$ & Bajas $^{\text {a }}$ \\
\hline D & 46 & 26,43 & 0 \\
\hline E & 26 & 14,94 & 0 \\
\hline F & 22 & 12,64 & 0 \\
\hline B & 17 & 9,77 & 2 \\
\hline G & 12 & 6,89 & 0 \\
\hline C & 14 & 8,04 & 0 \\
\hline A & 12 & 6,89 & 0 \\
\hline H & 174 & 100 & 2 \\
\hline Total & 18 & 4,02 & 0 \\
\hline
\end{tabular}

académico, y se rechaza la hipótesis nula en el sentido de que no existe una correlación significativa entre el nivel intelectual y el rendimiento académico previo, medido a través del promedio.

\section{Discusión}

De los factores socioacadémicos, destaca la tendencia predominante del género femenino sobre el masculino en la carrera de medicina. A nivel de licenciatura, la mayoría de los alumnos tiene red de apoyo familiar y social (profesión de los padres, número de hermanos, etc.) y estos factores no son tan determinantes en el rendimiento académico como en educación básica. Sólo el 9,7\% del alumnado es hijo de obreros y el $0 \%$ de campesinos, por lo que la movilidad social ascendente en este grupo es mínima (estructuralismo). El matrimonio y sus problemas en décadas pasadas eran una excepcionalidad, pero ahora la tendencia es que se recibe cada vez más a alumnos casados o con problemas de pareja (dos alumnas tuvieron que dejar el internado). Para conocer si esta condición afecta o no a su rendimiento aca- 
Tabla VI. Factores socioacadémicos de los médicos internos de pregrado, promoción 2005-2006, de diversas universidades.

\begin{tabular}{|c|c|c|c|c|}
\hline & & & Frecuencia & $\%$ \\
\hline \multirow{7}{*}{ Profesión del padre } & \multicolumn{2}{|l|}{ Profesional } & 94 & 54,02 \\
\hline & \multicolumn{2}{|l|}{ Funcionario } & 2 & 1,14 \\
\hline & \multicolumn{2}{|l|}{ Servicios } & 17 & 9,77 \\
\hline & \multicolumn{2}{|l|}{ Obrero } & 17 & 9,77 \\
\hline & \multicolumn{2}{|l|}{ Comercio } & 26 & 14,94 \\
\hline & \multicolumn{2}{|l|}{ Campesino } & 0 & 0 \\
\hline & \multicolumn{2}{|l|}{ Otros } & 18 & 10,34 \\
\hline \multirow{7}{*}{ Profesión de la madre } & \multicolumn{2}{|l|}{ Profesional } & 64 & 36,78 \\
\hline & \multicolumn{2}{|l|}{ Funcionaria } & 1 & 0,57 \\
\hline & \multicolumn{2}{|l|}{ Servicios } & 12 & 6,89 \\
\hline & \multicolumn{2}{|l|}{ Obrera } & 1 & 0,57 \\
\hline & \multicolumn{2}{|l|}{ Comercio } & 15 & 8,62 \\
\hline & \multicolumn{2}{|l|}{ Hogar } & 78 & 44,82 \\
\hline & \multicolumn{2}{|l|}{ Otros } & 3 & 1,72 \\
\hline \multirow{3}{*}{ Número de hermanos } & \multicolumn{2}{|l|}{$0-2$} & 98 & 56,32 \\
\hline & \multicolumn{2}{|l|}{$3-6$} & 68 & 39,08 \\
\hline & \multicolumn{2}{|l|}{$>6$} & 8 & 4,59 \\
\hline \multirow{4}{*}{ Estado civil } & \multicolumn{2}{|l|}{ Solteros } & 164 & 94,25 \\
\hline & \multicolumn{2}{|l|}{ Casados } & 7 & 4,02 \\
\hline & \multicolumn{2}{|l|}{ Unión libre } & 1 & 0,57 \\
\hline & \multicolumn{2}{|l|}{ Divorciados } & 2 & 1,14 \\
\hline \multirow{3}{*}{ Calificación más alta } & \multicolumn{2}{|c|}{ Materias teóricas } & 89 & 51,14 \\
\hline & \multicolumn{2}{|c|}{ Materias prácticas } & 58 & 33,33 \\
\hline & Materias socio & istas & 27 & 15,51 \\
\hline & Médica & & 85 & 48,85 \\
\hline Especialidad que piensan estudiar & Quirúrgica & & 87 & 50 \\
\hline & Otras & & 2 & 1,14 \\
\hline Promedio global & Máximo: 9,49 & Mínimo: 6,90 & Promed & $: 8,23$ \\
\hline & U. Gto & 8,74 & Desviació & ar: 0,38 \\
\hline & UVM & 8,71 & Desviació & ar: 0,18 \\
\hline & IPN & 8,62 & Desviació & ar: 0,34 \\
\hline & UAEH & 8,80 & Desviació & ar: 0,18 \\
\hline Promedio por universidad & UAQ & 8,40 & Desviació & ar: 0,25 \\
\hline & UAG & 8,05 & Desviació & ar: 0,28 \\
\hline & UMSH & 8,01 & Desviació & ar: 0,34 \\
\hline & UQI & 7,75 & Desviació & ar: 0,30 \\
\hline & ENEP-IZT & 7,51 & Desviació & ar: 0,19 \\
\hline
\end{tabular}




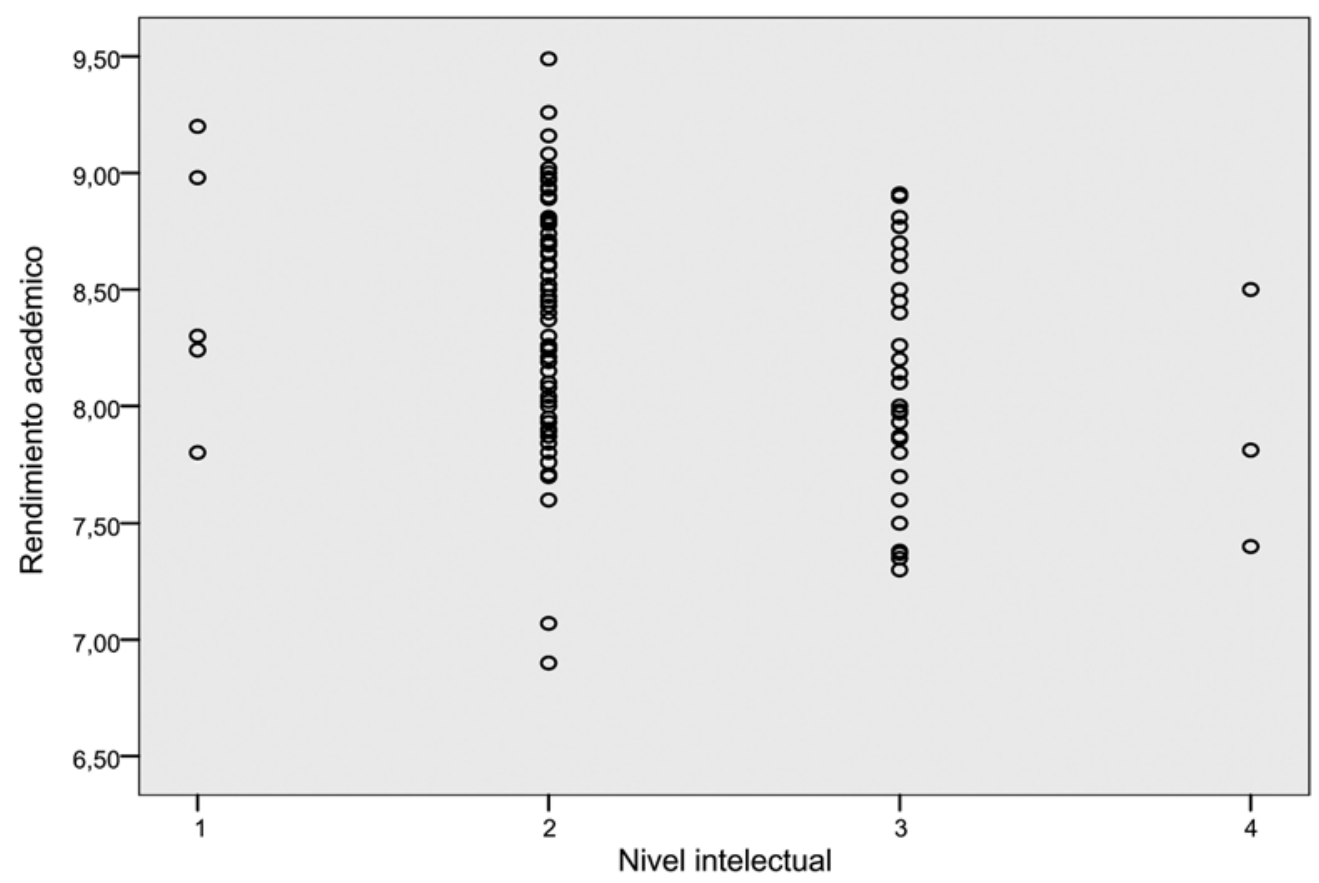

Figura 2. Gráfico de dispersión que muestra la relación del nivel intelectual con el rendimiento académico previo de los médicos internos de pregrado, promoción 2005-2006, de diversas universidades. 1: superior; 2: superior al término medio; 3: término medio; 4: inferior al término medio.

Tabla VII. Relación de factores socioacadémicos, estilos de aprendizaje y nivel intelectual con el rendimiento académico previo de los médicos internos de pregrado, promoción 2005-2006, de diversas universidades $(n=174)$.

\begin{tabular}{|c|c|c|c|}
\hline & Reg. mul. IC 95\% & Regresión $r$ & $p$ \\
\hline Género & $\chi^{2}=81,9$ & & $<0,176$ \\
\hline Estado civil & $\chi^{2}=6,64$ & & 1 \\
\hline Universidad de procedencia & $\chi^{2}=173$ & & 1 \\
\hline Profesión del padre & $\chi^{2}=60,5$ & & 1 \\
\hline Profesión de la madre & $\chi^{2}=57$ & & 1 \\
\hline Número de hermanos & & $-0,002$ & 0,49 \\
\hline Edad & & 0,2 & $<0,001^{a}$ \\
\hline Nivel intelectual & & 5,95 & $<0,001^{\mathrm{a}}$ \\
\hline Estilos de aprendizaje & $\chi^{2}=34$ & & 1 \\
\hline
\end{tabular}


Tabla VIII. Preferencia por estilos de aprendizaje de los médicos internos de pregrado, promoción 2005-2006, de diversas universidades.

\begin{tabular}{lcc}
\hline & Frecuencia & $\%$ \\
\hline Teórico & 96 & 55,17 \\
\hline Pragmático & 38 & 21,83 \\
\hline Activo & 20 & 11,49 \\
\hline Reflexivo & 18 & 10,34 \\
\hline Perdidos & 2 & 1,14 \\
\hline Total & 174 & 100 \\
\hline
\end{tabular}

démico futuro, serán precisos estudios longitudinales o de cohortes. Por otra parte, el reglamento vigente de los MIP no contempla el manejo administrativo de situaciones como incapacidad por maternidad, lactancia, etc. La clasificación de asignaturas en predominantemente teóricas, prácticas y sociohumanistas es artificial, pero permite, hasta cierto punto, corroborar lo que observan la mayoría de los profesores con los que rotan los alumnos: la deficiencia con la que llegan los alumnos al internado en áreas sociohumanistas, particularmente en clínica y bioética. Con excepción de dos alumnos, la abrumadora mayoría desearía estudiar una especialidad, y seleccionaría por igual especialidades clínicas o quirúrgicas, con los efectos que ya conocemos: sólo unos cuantos llegarán, lo que ocasionará frustración, desempleo, subempleo, etc. La única variable socioacadémica que tuvo una relación inversa con el rendimiento académico fue la edad. Esto se explica por el rango de edad de nuestra población, lo que no sucede en ciclos previos, por ser grupos más homogéneos. Una de las limitaciones de nuestra encuesta es que no incluimos preguntas relacionadas con consumo de tabaco, alcohol o drogas, o violencia intrafamiliar, ya que generan ansiedad y sesgo de respuesta.

El promedio con el que llegan los alumnos es bueno, aunque excesivamente disperso, con un
Tabla IX. Nivel intelectual de médicos internos de pregrado, promoción 2005-2006, de diversas universidades.

\begin{tabular}{lcc}
\hline & Frecuencia & $\%$ \\
\hline Superior & 5 & 2,87 \\
\hline Superior al término medio & 106 & 60,91 \\
\hline Término medio & 58 & 33,33 \\
\hline Inferior al término medio & 3 & 1,72 \\
\hline Perdidos & 2 & 1,14 \\
\hline Total & 174 & 100 \\
\hline
\end{tabular}

rango amplio de 6,9 a 9,4 y una DE de 0,49. Esto tiene su explicación, entre otros factores, en que los MIP seleccionan sede de internado de pregrado de acuerdo con su promedio y, lógicamente, seleccionan, en primer lugar, aquellas plazas más cercanas a su lugar de residencia.

Evaluar el rendimiento académico o desempeño es bastante más complejo que medirlo a través del promedio, que, además, traduce predominantemente la capacidad de retención o memoria por parte del alumno. Éste es el modelo educativo tradicional o pasivo-receptivo [13]. Pero así es como se evalúa en la vida real en la mayoría de escuelas. La situación se complica, porque no todas las universidades califican con el mismo rigor o criterio a los alumnos. Este estado de cosas no es privativo de las escuelas de medicina o internado de pregrado o posgrado, sino también de escenarios hospitalarios [14,15].

Sackett et al consideran que para evaluar el desempeño se debe tomar en cuenta no sólo los conocimientos, habilidades y actitudes, sino sumar la motivación para aplicarlos y restar los obstáculos de los escenarios clínicos y, algo muy importante, su retroalimentación a través de la evaluación sistemática [16]. Sin embargo, aunque se ha avanzado en este terreno, en el internado y en posgrado su aplicación dista de generalizarse, entre otras razones por falta de estructura docen- 
te o formación y actualización de los profesores en las nuevas tendencias educativas (elaboración del conocimiento, aprendizaje basado en problemas, examen clínico objetivo estructurado, medicina basada en la evidencia, etc.) [17-19]. En los ciclos básicos, el instrumento de evaluación por excelencia son los exámenes tradicionales o de opción múltiple. De ahí la necesidad de validar instrumentos que evalúen la formación de los médicos desde ciclos básicos y posgrado para que dicha valoración sea más congruente y apegada a la realidad social con la que se enfrentará el futuro médico [20].

El estilo de aprendizaje (estilo cognitivo o estilo motivacional, etc.) de la mayoría de los alumnos es teórico (su 'fortaleza'), y el menos preferido es el estilo reflexivo (su 'debilidad'), sin diferencias entre géneros o escuela de procedencia. Este estudio corrobora la teoría de que la mayoría de los estudiantes tiene, en mayor o menor proporción, preferencia por un estilo (fortaleza y debilidad respectivamente). Conocerlo permitiría explotar su propio estilo y explorar y ejercitar otros para mejorar su rendimiento académico, pero se requieren estudios de intervención para comprobarlo. Se ha demostrado que en residentes de cirugía predomina el estilo pragmático, de tal forma que conocer su estilo de aprendizaje permitiría orientar al alumno en la selección de una especialidad [21]. Algunos autores proponen la aplicación de todos los estilos de aprendizaje en forma secuencial en encuentros educacionales [22]. En nuestro trabajo no encontramos asociación entre el estilo de aprendizaje del alumno con su rendimiento académico medido por el promedio, lo que sugiere que éste tampoco es una buena medida del aprendizaje experiencial o elaboración del conocimiento; habrá que recurrir a otras formas de evaluar el rendimiento para poder establecer con mayor certeza si el estilo se asocia o no con el rendimiento académico.

El test de matrices progresivas de Raven es un instrumento de evaluación particularmente útil en investigación médica-educacional. Su aplicación y evaluación es sencilla y no se requiere preparación especial o experiencia previa, se puede aplicar a todo sujeto de cualquier edad, educación, idioma y aptitud motriz, es económico y se autoadministra o se aplica colectivamente; además, es independiente del factor cultural. El test busca medir el factor general $(G)$ o capacidad intelectual general en algunas funciones cognoscitivas, como la observación y el razonamiento. Como era de esperar, en nuestro estudio encontramos que la variable inteligencia tuvo una curva de distribución normal. Lo que no esperábamos, dada nuestra hipótesis, es su asociación en el rendimiento. Esta asociación es clara en grupos menores de 18 años, donde los factores genéticos, ambientales y nivel socioeconómico son determinantes para el rendimiento [23]. Pero en adultos esta relación es incierta. Estos resultados, lógicamente, no se pueden extrapolar a otros grupos de MIP y menos aún generalizarse, son aplicables sólo a los grupos estudiados en esta muestra.

\section{Bibliografía}

1. Beguet B, Cortada de Kohan N, Castro A, Renault G. Factores que intervienen en el rendimiento académico de los estudiantes de psicología y psicopedagogía. Revista Científica de la Dirección de Evaluación y Acreditación de la Secretaría General de la Universidad del Salvador-USAL 2001; 1 .

2. Alonso C, Gallego D, Honey P. Los estilos de aprendizaje. 4 ed. Bilbao: Mensajero; 1999.

3. Pozo J. Teorías cognitivas del aprendizaje. 5 ed. Madrid: Morata; 1997.

4. Gardner H. Mentes flexibles. México: Paidós; 2005.

5. Faure E, Herrera F, Kaddoura AR, Lopes H, Petrovski AV, Rahmena M, et al. Aprender a ser, la educación del futuro. 3 ed. Madrid: Alianza-UNESCO; 1974.

6. Kolb A, Kolb DA. Learning styles and learning spaces: enhancing learning in higher education. Acad Manag Learn Educ 2005, 4: 193-212.

7. Reese AC. Implications of results from cognitive science research for medical education. Med Educ Online 1998; 3: 1-9.

8. Martín-García AV. Estilos de aprendizaje en la vejez. Un estudio a la luz de la teoría del aprendizaje experiencial. Rev Esp Geriatr Gerontol 2003; 38: 258-65.

9. Raven JC. Court JH, Raven J. Test de matrices progresivas. Buenos Aires: Paidós; 1993.

10. Viniegra-Velázquez L. Las enfermedades crónicas y la educación. Rev Med Inst Mex Seguro Soc 2006; 44: 47-59.

11. Canalejas P, Martínez M, Pineda G, Vera C, Soto G, Martín $\mathrm{M}$, et al. Estilos de aprendizaje en los estudiantes de enfermería. Educ Med 2005; 8: 83-8.

12. Alonso C, Gallego D, Honey P. CHAEA: cuestionario 
Honey-Alonso de estilos de aprendizaje. 4 ed. Bilbao: Mensajero; 1999.

13. Viniegra-Velázquez L, Jiménez JL, Pérez-Padilla JR. El desafío de la evaluación en la competencia clínica. Rev Invest Clín 1991; 43: 87-95.

14. González-Cobos RP, Viniegra-Velázquez L. Comparación de dos intervenciones educativas en la formación de médicos residentes. Rev Invest Clin 1999; 51: 351-60.

15. García-Mangas JA, Viniegra-Velázquez L, Arellano-López J, García-Moreno J. Evaluación de la aptitud clínica en médicos del primer nivel de atención. Rev Med Inst Mex Seguro Soc 2005; 43: 465-72.

16. Sackett $D$. Introducción: revisión del propio desempeño. In Sackett D, Haynes R, Guyatt G, Tugwell P, eds. Epidemiología clínica. 2 ed. Buenos Aires: Panamericana; 1994. p. 301-26.

17. De Serdio-Romero E. ECOE: evaluación clínica objetiva estructurada. Medicina de Familia (And) 2002; 3: 127-32.

18. Trejo-Mejía J, Estrada D, Peña J, Garnica J, Ángeles R, Díaz J, et al. Desafíos de la enseñanza de la medicina fa- miliar en el pregrado: el caso de México. Arch Med Fam 2005: 7: 109-16.

19. Universidad Michoacana de San Nicolás de Hidalgo. Manual del médico interno de pregrado 2005-2006. México: Facultad de Medicina Dr. Ignacio Chávez.

20. Viniegra-Velázquez L. La formación de especialistas en el Instituto Mexicano del Seguro Social. Hacia un nuevo sistema de evaluación. Rev Med IMSS 2005; 43: 141-53.

21. Contessa J, Ciardiello KA, Perlman S. Surgery resident learning styles and academic achievement. Curr Surg 2005; 62: 344-7.

22. Armstrong E, Parsa-Parsi R. How can physicians' learning styles drive educational planning? Acad Med 2005; 80: 680-4.

23. Ivanovic-Marincovich R, Forno-Sparosvich H, DuránSantana MC, Hazbún-Game J, Castro-Gómez C, Ivanovic-Marincovich D. Estudio de la capacidad intelectual (test de matrices progresivas de Raven) en escolares chilenos de 5 a 18 años. Revista de Psicología General y Aplicada 2000; 53: 5-30. 